\title{
Thermoacoustic Refrigerator for High Temperature Gradient
}

\author{
Abhishek Mishra ${ }^{1}$, Ankur Kumar Choudhary ${ }^{1}$, Taniya Tomar $^{1}$ and Jagannath Korody ${ }^{1 *}$ \\ ${ }^{1}$ Manipal Institute of Technology, Manipal Academy of Higher Education, Manipal, Karnataka, India
}

\begin{abstract}
Leakage of HFCs, thermal shocks to sensitive devices, moving mechanical components which in turn require more maintenance, are some of the cons of a conventional vapor compression system .The newest field in refrigeration overcoming such flaws is Thermoacoustic refrigerator (TAR), where the sound energy is used to generate a temperature difference which can be used for refrigeration. TAR are environment friendly, do not use any kind of refrigerants, and as the compressor and expander are not used, hence, its life is more than a conventional VCR. The objective is to obtain a substantial temperature gradient using a Thermoacoustic Refrigerator. The methodology is to choose the suitable material and design the prototype. A large part of this project is devoted to explore the different designs for thermo acoustic refrigerators, and select the optimum one for maximum utility. With the present system, it was possible to achieve a temperature difference across the stack of $14 \mathrm{~K}$.
\end{abstract}

\section{Introduction}

The basic working of the Heat engines and refrigerators can be explained by the laws of thermodynamics. A commonly used Vapor Compression Refrigeration Cycle which consists of 4 units; Compressor, Condenser, Expander or a Throttle Valve (A small capillary tube is generally used) and an Evaporator. The problem with VCRs is the power required by the Compressor is high, wear \& tear of the compressor as it has moving parts and also the leakage of refrigerant can be hazardous. Therefore there is a need to go for alternative refrigeration concepts. One of the concepts that can be used is Acoustics. Thermoacoustic Refrigerator is such a concept which uses sound to create a refrigeration effect. So the compressor and the expander processes of a conventional VCR are replaced by the sound sources which have compression \& rarefaction zones. In the compression zones the temperature is high and in the rarefaction zone the temperature is low. Thus, this concept is used to create a refrigeration effect. Thermoacoustic Refrigerators are environment friendly as the working medium chosen is air.

\subsection{Literature Review}

The performance of Thermoacoustic Refrigerator depends upon the different stack geometry i.e. spiral stack, parallel plate stack or pin array stack. It also depends upon the working medium inside the resonating tube i.e. He, Air, He-Xe mixture, hydrogen, etc. The selection

\footnotetext{
*Corresponding author: jagan.korody@manipal.edu
} 
of materials for the resonating tube and the stack plays a vital role in the performance of Thermoacoustic Refrigerator. Some of the materials suitable are Kapton, Mylar, Polymer Film, Plexi glass, PVC glass, and acrylic fiber. One of the literatures discuss about the behavior of Standing wave TAR regarding the COP. The COP of the TAR is generally low. The Thermoacoustic Refrigeration has a linear relationship with frequency and thus high operating frequency is desired. A higher operating frequency results in a small stack plate spacing but makes the construction difficult. The position of the stack from the driver end plays a vital role in the performance of Thermoacoustic Refrigerators hence it is desired to find an optimum position for the stack. It also depends upon the porosity of the stack. According to various literatures it is desirable to use quarter wavelength resonator over half wavelength resonator because it dissipates only half the energy compared to half wavelength resonator. Hence the minimization of the energy loss can be used to make the device compact and efficient.

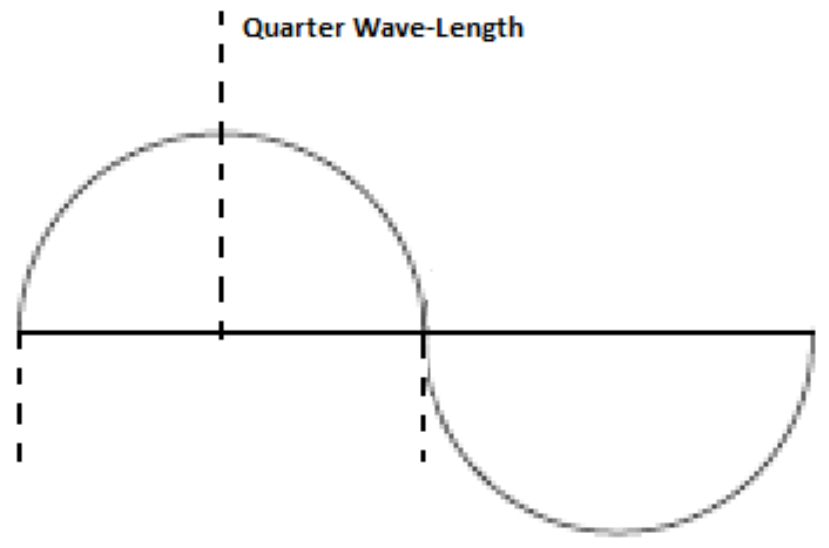

Fig. 1. Quarter Wave Length Representation

\section{Working of the system}

A Thermoacoustic refrigerator consists of generally a Speaker, an Amplifier, a Resonating Tube, a Stack, and a Speaker Casing. The speaker is used to create sound waves which can be monitored at a certain frequency. The amplifier is used to vary the amplitude of the sound waves. Resonating tube (Resonator) consists of the working medium and encases the stack. It is the resonator in which the whole working takes place. A stack is the heart of the Thermoacoustic refrigeration which is one of the most crucial components for having the cooling effect. A sound wave compresses and expands at different regions in the resonator. A stack is located at a certain position inside the resonator. The sound wave absorbs heat from the cooler region and pumps it to the stack. The next sound parcel takes the heat from that layer of the stack and passes it to the next adjacent layer of the stack. This step continues and finally the heat is transferred from the cooler region to hot region and thus the cooler region becomes cooler and the hotter region becomes hotter. A heat exchanger can also be used to aid the performance of the Thermoacoustic refrigerator.

The Thermoacoustic refrigerator demonstration is of a standing wave type and consists of a quarter wavelength resonator driven by a speaker. In the apparatus demonstration an 860 $\mathrm{W}$ speaker is used. The primary purpose of the refrigerator is to demonstrate the Thermoacoustic refrigeration effect and hence the efficiency was not our primary concern. 
The amplifier used was Flyball IPL-108 of low capacity to change the amplitude of the sound waves. The setup is shown in the figure below

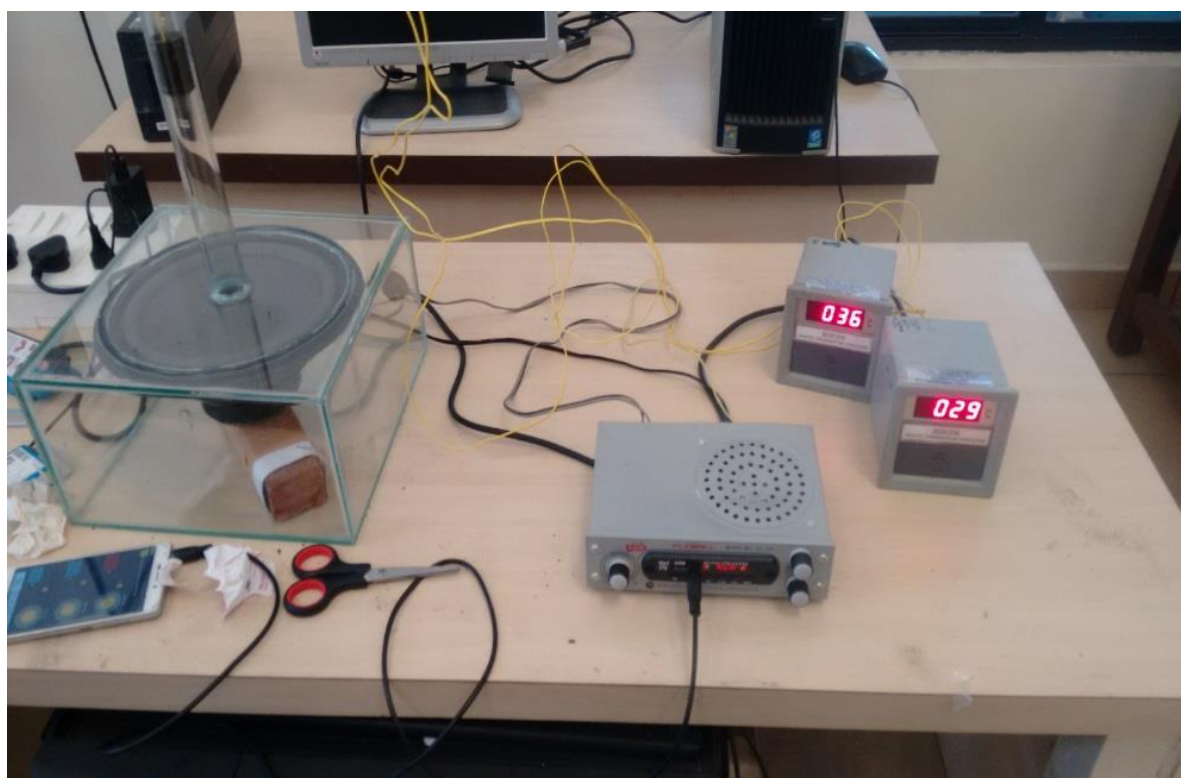

Fig. 2. Experimental setup for the TAR

The resonator for this refrigerator was a $22 \mathrm{~cm}$ length of glass tube with inner diameter $2.2 \mathrm{~cm}$. The length defines the resonant frequency of the system as $379 \mathrm{~Hz}$ for our apparatus. Frequency generator was used to vary the frequency. A glass casing of glass thickness $4 \mathrm{~mm}$ was made with dimension 10 X 10 X 5 inches. A One inch hole was cut on the top face of the glass casing, and one end of the resonating tube was placed on top of the hole with central axis aligned. The glass tube was adhered to the glass box and sealed properly with silicon resin as the adhesive. Two O-ring of 9 inch diameter were chosen to avoid leakage and seal properly the speaker to the surface of the glass casing top surface from the inner side. A plug made of mild steel was used to seal the resonator from the top end and a hole was drilled at the center of the plug with the help of a $5.5 \mathrm{~mm}$ drill bit on a vertical drilling machine. Then the facing operation of the plug was done on an engine lathe to obtain high surface finish. This hole was drilled to pass the thermocouple wires through it.

Stack material should have a low thermal conductivity and a higher thermal capacity than the working medium. The stack was made from a $35 \mathrm{~mm}$ photographic film. The stack geometry used was of Spiral (rolled) type. The spacing between the adjacent layers of the stack is filled by the nylon fishing line $(0.50 \mathrm{~mm}$ thick $)$. Fishing lines were stuck onto the surface of the sheet with the help of a polymer tape and then spirally rolled, being aligned parallel to the length of the resonator. The spacing between the linings has to be optimum; if the layers are close together viscous effects hamper the motion of the gas particles and if the layers are too wide apart the heat transfer rate would not be much due to leakage of air across the gaps.

Thermocouple of K-type was used. The wires of the thermocouple passed through the hole of the plug and measured the temperature at the two ends of the stack. The important factor while placing the thermocouple wires was that they should not touch the stack surface but they should be located as closely as possible to the stack. Two displays for the thermocouples were used to indicate the temperature. Sealing of the whole system was done with silicon resin and M-seal. 
Thermal penetration gap is given by the expression

$\delta=\sqrt{(2 K} / \rho c \omega)$

$\mathrm{K}=$ Gas thermal conductivity

$\rho=$ Density

$\mathrm{c}=$ Isobaric Specific Heat

$\omega=$ Operating frequency in radians per second

Therefore thermal penetration gap is calculated to be $1.30 \times 10^{-5} \mathrm{~m}$ or $0.013 \mathrm{~mm}$.

\section{Results and Discussion}

Testing of the apparatus was done at various frequencies ranging from $345-385 \mathrm{~Hz}$. Also the position of the stack was varied to obtain various results.

Initially the testing was done with a frequency of $385 \mathrm{~Hz}$ and stack position of $5 \mathrm{~cm}$ from the top (plug surface). The setup ran for 12 minutes and a temperature gradient of 2 Kelvin was noted along with 1 Kelvin of cooling. With a frequency of $379 \mathrm{~Hz}$ and stack position of $4 \mathrm{~cm}$ from the top, the trial resulted in a gradient of 4 Kelvin and cooling of 1 Kelvin. With a frequency of $374 \mathrm{~Hz}$ and a stack position of $3 \mathrm{~cm}$ from the top, the gradient obtained was 5 Kelvin with 2 Kelvin of cooling. With a frequency of $369 \mathrm{~Hz}$ and a stack position $2 \mathrm{~cm}$ from the top, a gradient of 8 Kelvin with 3 Kelvin of cooling was observed.

Finally a frequency of $364 \mathrm{~Hz}$ showed the best result with the stack being placed at $1 \mathrm{~cm}$ from the top which resulted in a thermal gradient of 14 Kelvin with 5 Kelvin of cooling in just 33 minutes, i.e. the hot side of the stack rose up by $9 \mathrm{~K}$ and the cooler side cooled down by $5 \mathrm{~K}$. It can be noted from the Fig. 2 that the variation of the temperature is not linear with the time.

Figure 4 shows that after some period of time (in minutes) the temperature-time behaviors lies near to the straight line where as in during the initial minutes there is a deviation from the straight line. Also the temperature gradient is very rapid during the initial few minutes of the run of the apparatus but very slow afterwards.

Table 1. Variation of Temperature for $\mathrm{f}=364 \mathrm{~Hz}$

\begin{tabular}{|l|l|l|l|}
\hline Time $(\mathrm{min})$ & Temperature Gradient & $\mathrm{T}_{1}{ }^{\circ} \mathrm{C}(\mathrm{K})$ & $\mathrm{T}_{2}{ }^{\circ} \mathrm{C}(\mathrm{K})$ \\
\hline 0 & 0 & $30(303)$ & $30(303)$ \\
\hline 1 & 4 & $32(305)$ & $28(301)$ \\
\hline 2 & 6 & $34(307)$ & $28(301)$ \\
\hline 3 & 7 & $34(307)$ & $27(300)$ \\
\hline 4 & 8 & $35(308)$ & $27(300)$ \\
\hline 8 & 10 & $37(310)$ & $27(300)$ \\
\hline 15 & 11 & $38(311)$ & $27(300)$ \\
\hline 22 & 12 & $38(311)$ & $26(299)$ \\
\hline 27 & 13 & $39(312)$ & $26(299)$ \\
\hline 33 & 14 & $39(312)$ & $25(298)$ \\
\hline
\end{tabular}




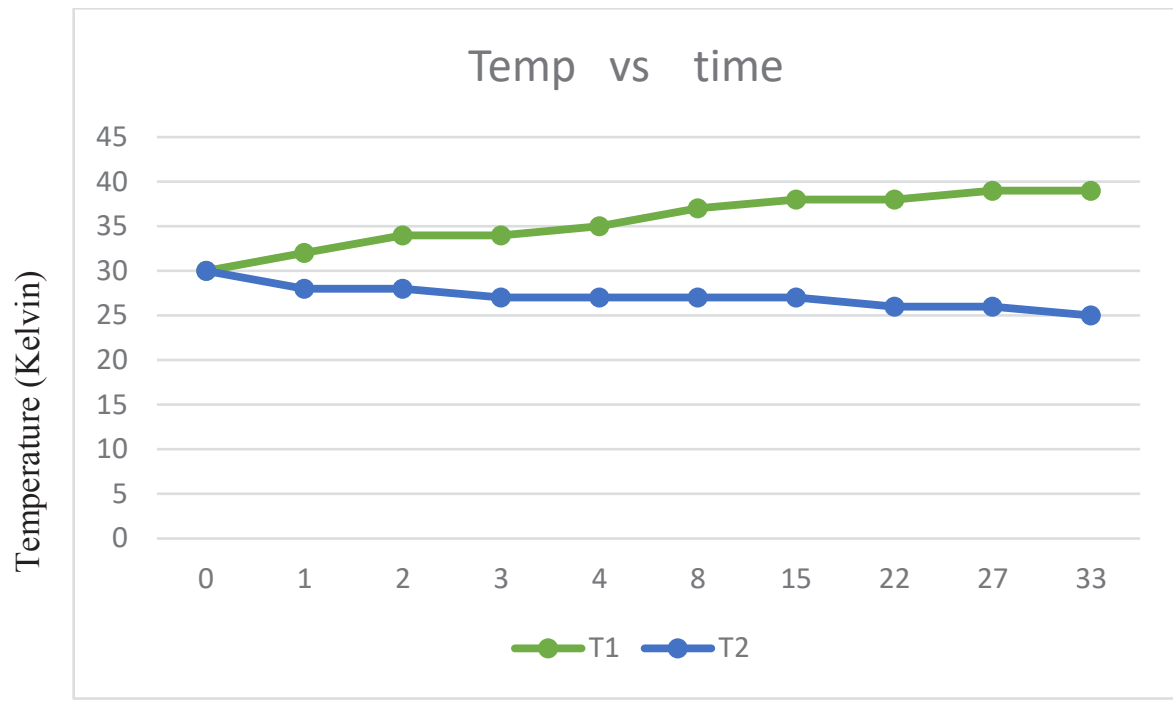

Time (minutes)

Fig. 3. Plot of variation of Hot and Cold Region across a stack with respect to time

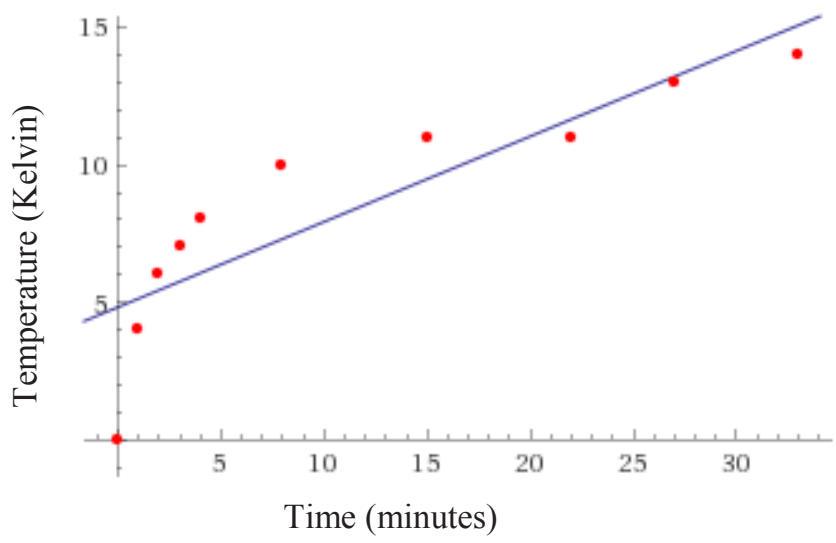

Fig. 4. Scatter plot for the Temperature Gradient

\section{Conclusions}

The results from the experiment show that a significantly high amount of Temperature gradient can only be achieved when all the significant parameters are well taken care of. Hence we conclude from the trials that:

i. The maximum temperature gradient obtained was $14 \mathrm{~K}$, with the maximum drop in colder region temperature being $5 \mathrm{~K}$, when the stack was placed at its most optimum position and the setup was operated with the most optimum frequency along with proper sealing which is quite good for a basic Thermoacoustic refrigerator model. The testing setup was started at an initial temperature of $303 \mathrm{~K}$. The hotter thermocouple went on to reach a temperature of $312 \mathrm{~K}$, i.e. a rise of $9 \mathrm{~K}$, whereas the cold thermocouple showed 
a drop in temperature of $5 \mathrm{~K}$ and thus read $298 \mathrm{~K}$ after the setup was run for about 33 minutes.

ii. The most efficient stack position was $1 \mathrm{~cm}$ from the top (distance measured from the bottom of the Mild Steel Plug). The optimum stack position is very critical in obtaining the best possible or the highest achievable thermal gradient which depends on the geometry of the resonator and also on the frequency of the sound waves. A temperature difference of $14 \mathrm{~K}$ was obtained when the stack was placed $1 \mathrm{~cm}$ from the top of the resonator tube.

iii. The most optimum frequency was found to be $364 \mathrm{~Hz}$. The calculations according to the length of the resonating tube and stack position resulted in a frequency of $369 \mathrm{~Hz}$, but by hit and trial it came to 364 during the testing phase. We hence concluded that there are few unaccounted losses in transmission of sound waves which resulted in the deviation from the ideal calculations, owing to friction due to glass surface, viscous damping effects of air, and also maybe because of the thermal penetration depth of the stack layers were not perfectly uniform.

iv. Very less or almost zero temperature gradient will be obtained even if there is a slight leak in the setup, and hence for maximum efficiency of the apparatus it has to be hermetically sealed.

v. Stack being the most important part of the apparatus has to be rolled very carefully. If it is rolled too tight then viscous damping effects dominate which lead to loss in energy transfer and if it is rolled too loosely then the air particles tend to escape through the gaps without any heat exchange with the stack surfaces.

vi. Thermocouples are used to measure the air temperature and not the stack surface temperature.

vii. Use of heat exchangers can significantly increase the thermal gradient as it will accelerate the heat exchange process across the stack.

viii. Higher the power rating of the speaker, higher is the maximum amplitude of the sound wave, and hence higher the amount of thermal gradient that can be achieved.

ix. Use of pressurized air or lighter gases like helium can give better results but involve costlier setups and more complicated designs.

x. Stack material should have low thermal conductivity and higher thermal capacity than the working medium.

xi. The power density of the Thermoacoustic refrigerator is a linear function of the frequency, and hence high value of frequency is desirable. But high frequency results in a small stack plate spacing and that makes construction difficult. Hence the selection of the optimum frequency is required, which is obtained using a suitable loudspeaker.

\section{References}

1. Timothy S.Ryan B.S in Mechanical engineering, "Design and Control of a StandingWave Thermoacoustic Refrigerator", University of Pittsburg, (2006)

2. M.E.H. Tijani, J.C.H Zeegers, A.T.A.M de waele "Design of Thermoacoustic refrigerators", - Elsevier Science, (2002).

3. Ratish Savant, Sandesh Rasal, Gaurav Yadav, Arunkumar Yadav, Darshan Kadam , "Design \& Fabrication of Thermoacoustic Refrigeration", International Journal of Research in Advent Technology, Vol.3 , No.4,April (2015)

4. Ratish Savant, Sandesh Rasal, Gaurav Yadav, Arunkumar Yadav, Darshan Kadam , "Design \& Fabrication of Thermoacoustic Refrigeration", International Journal of Research in Advent Technology, Vol.3, No.4,April (2015) 
5. Jinshah B S, Ajith Krishnan R, Sandeep V S ,"Study on A Standing Wave Thermoacoustic Refrigerator Made of Readily Available Materials" International Journal of Scientific and Research Publication, Volume 3, Issue, July (2013)

6. B.G Prasantha, M.S.Govinde Gowda, S. Sreetharamu , "Effect of mean operating pressure of the performance of Stack Based Thermoacoustic Refrigerator" International Journal of Thermal \& Environment Engineering, Volume 5, (2013) 